\title{
Finite-Time Boundedness Analysis for a Class of Switched Linear Systems with Time-Varying Delay
}

\author{
Yanke Zhong and Tefang Chen \\ School of Information Science and Engineering, Central South University, Changsha 410075, China \\ Correspondence should be addressed to Yanke Zhong; zhongyanke1981@163.com
}

Received 21 October 2013; Accepted 1 January 2014; Published 13 February 2014

Academic Editor: Valery Y. Glizer

Copyright ( 2014 Y. Zhong and T. Chen. This is an open access article distributed under the Creative Commons Attribution License, which permits unrestricted use, distribution, and reproduction in any medium, provided the original work is properly cited.

The problem of finite-time boundedness for a class of switched linear systems with time-varying delay and external disturbance is investigated. First of all, the multiply Lyapunov function of the system is constructed. Then, based on the Jensen inequality approach and the average dwell time method, the sufficient conditions which guarantee the system is finite-time bounded are given. Finally, an example is employed to verify the validity of the proposed method.

\section{Introduction}

The switched system is a special kind of hybrid dynamic system, composed of a family of subsystems and a switching law specifying the switches between subsystems $[1,2]$. The fact that the structure and working mechanism of the switched system are more complex than general systems leads to that the switched system possesses much richer dynamic characteristics. The switched systems are widely applied in engineering practice, such as power system control, robot control, network control, and so forth [3-9].

In practice, switched systems are commonly subjected to time-delay and external disturbance. Due to their significant impact on the performances of switched systems, many scholars have been attracted to investigate the problem. Sun et al. analyzed the asymptotic stability of the switched linear system with time-delay perturbation by using common Lyapunov function and multiple Lyapunov function [3]. $\mathrm{Lu}$ and Zhao also investigated the asymptotic stability for switched linear systems with time-delay and proposed an effective method which can direct researchers to choose an appropriate switching law to make sure the system is asymptotic stable [10]. Zhao and Zhang studied the stability of the switched system with time-varying delays based on the average dwell time and time-delay decomposition approaches [11]. For switched systems with time-varying delay, Lian et al. utilized the Lyapunov-Krasovskii function method to design $\mathrm{H}$ infinity filter [12]. For switched systems affected by the nonlinear impact and disturbance, Sun used transfer matrix estimation and Gronwall inequality methods to design a feedback law stabilizing system [13]. For the switched system with fixed time-delay and norm bounded disturbance, Lin et al. proposed the finite-time boundedness concept and a method to judge whether the system is finite-time bounded [14].

Up to now, to the best of the authors' knowledge, there are a few papers concerning the finite-time boundedness problem of switched system. For switched systems with timevarying delay and external disturbance, the problem has not yet been discussed by any literature. However, in practical engineering, the time-delays are generally changeable over time, not fixed. In addition, many practical systems are just required that their state trajectories are bounded over a fixed interval. In other words, those systems may be unstable. On the contrary, although some systems are asymptotically stable, they cannot meet the application requirements because of their large transient state amplitudes. Considering the wide application of switched systems with time-varying delay and the requirements for transient behaviors in engineering fields, it is a significant task to investigate finite-time boundedness for switched linear systems with time-varying delay and external disturbance. The main contributions in this paper are 
listed as follows. (1) For the convenience of processing, a concise definition on the finite-time boundedness is proposed for the switched system. (2) Sufficient conditions of finite-time boundedness for switched linear systems with time-varying delay and external disturbance are given.

\section{Preliminaries and Problem Formulation}

Consider the following switched linear system with timevarying delay and external disturbance:

$$
\begin{gathered}
\dot{x}(t)=A_{\sigma(t)} x(t)+B_{\sigma(t)} x(t-h(t)) \\
\quad+G_{\sigma(t)} w(t), \quad h(t) \geq 0, \quad t \geq 0, \\
x(t)=\varphi(t), \quad \max |\dot{\varphi}(t)| \leq \rho, \quad \rho \geq 0, \\
\quad t \in[-d, 0), \quad d \geq h(0),
\end{gathered}
$$

where $x(t)$ is state variable and $\sigma(t)$ is the switching law which is a piecewise continuous function with $\sigma(t) \in M=$ $[1,2, \ldots, m]$ which means the switched system is consisted of $m$ subsystems. The $i$ th subsystem is activated when $\sigma(t)=$ $i \cdot A_{\sigma(t)}, B_{\sigma(t)}$, and $G_{\sigma(t)}$ are constant matrices. $h(t)$ represents time-varying delay. $w(t)$ stands for external disturbance. $\varphi(t)$ is the continuous vector-valued initial function on $t \in[-d, 0) \cdot \dot{\varphi}(t)$ denotes the derivative of $\varphi(t) \cdot \rho$ is a positive constant.

For the convenience of subsequent processing, assume that the system (1) satisfies the following assumptions.

Assumption 1 (see [14]). The value of external disturbance changes over time, but it satisfies

$$
\int_{0}^{+\infty} w^{T}(t) w(t) d t \leq \gamma, \quad \gamma \geq 0, \forall t>0
$$

Assumption 2. For the time-varying delay, the following inequalities hold:

$$
h(t) \geq 0, \quad \dot{h}(t) \leq k, \quad k<1, \quad h(t) \leq h_{\max }
$$

where $k$ and $h_{\max }$ are positive constants.

Assumption 3. The system state variable does not "jump" at switching instant, that is to say the state trajectory is continuous. In addition, the switching number of $\sigma(t)$ is finite in a limited time interval which implies that the frequency of switching signal is not infinite.

Definition 4 (see [15]). For $T \geq t \geq 0$, let $N_{\sigma}(t, T)$ denote the switching number of $\sigma(t)$ over $(t, T]$. If

$$
N_{\sigma}(t, T) \leq N_{0}+\frac{T-t}{\tau_{a}}
$$

holds for $\tau_{a} \geq 0$ and an integer $N_{0} \geq 0$, then $\tau_{a}$ is called average dwell time.

Definition 5. For a given four positive constants $c_{1}, c_{2}, T_{f}, \gamma$, and a switching signal $\sigma(t)$, if

$$
\begin{array}{r}
x^{T}\left(\bar{t}_{0}\right) x\left(\bar{t}_{0}\right) \leq c_{1} \Longrightarrow x^{T}(t) x(t)<c_{2}, \\
c_{1}<c_{2}, \forall t \in\left[0, T_{f}\right], \\
\forall w(t): \int_{0}^{T_{f}} w^{T}(s) w(s) d t \leq \gamma,
\end{array}
$$

then the system (1) is said to be finite-time bounded. Where $x^{T}\left(\bar{t}_{0}\right) x\left(\bar{t}_{0}\right)=\sup _{-d \leq t \leq 0}\left\{x^{T}(t) x(t)\right\}$, without loss of generality, specify $c_{1}=\sup _{-d \leq t \leq 0}\left\{x^{T}(t) x(t)\right\}$.

Remark 6. Definition 5 implies that if the system (1) is finitetime bounded, the state remains within the prescribed bound in the fixed interval. Notice that finite-time boundedness is different from asymptotic stability. The system which is finite-time bounded may not be asymptotically stable while a system is asymptotically stable does not mean it is finitetime bounded either. In a word, there is no necessary relation between them.

Remark 7. The definition of finite-time boundedness in this paper is much more concise than that in [14]. However, they are consistent in essence. By using the definition in this paper, some complex matrix transformations can be avoided in the subsequent mathematical processing.

\section{Main Result}

Theorem 8. For system (1), for all $i \in M$ and for all $t \in\left[0, T_{f}\right]$, assume there exists symmetric positive matrixes $P_{i}, R_{i 1}, R_{i 2}, Q_{i}, Z_{i 1}, Z_{i 2}$, and $H$ and positive constants $\alpha, \beta \geq 1$ such that

$$
\left[\begin{array}{ccccc}
\xi_{11} & P_{i} B_{i}+\frac{d}{2} A_{i}^{T} Z_{i} B_{i} & \frac{2}{d} e^{\alpha d / 2} Z_{i, 1} & 0 & P_{i} G_{i}+\frac{d}{2} A_{i}^{T} Z_{i} G_{i} \\
* & \frac{d}{2} B_{i}^{T} Z_{i} B_{i}-(1-k) Q & 0 & 0 & \frac{d}{2} B_{i}^{T} Z_{i} G_{i} \\
* & * & e^{\alpha d / 2} R_{i}-\frac{2}{d} e^{\alpha d / 2} Z_{i} & \frac{2}{d} e^{\alpha d} Z_{i, 2} & 0 \\
* & * & * & -e^{\alpha d} R_{i, 2}-\frac{2}{d} e^{\alpha d} Z_{i, 2} & 0 \\
* & * & * & * & \frac{d}{2}\left(G_{i}^{T} Z_{i} G_{i}-H\right)
\end{array}\right]<0 .
$$


If the average dwell time satisfies

$$
\tau_{a}<\frac{T_{f} \ln \beta}{\ln C_{1}+\ln \lambda_{8}-\ln \left(\eta_{1} C_{1}+\eta_{2}\right)-\alpha T_{f}},
$$

then system (1) is finite-time bounded, where

$$
\begin{aligned}
& \xi_{11}=A_{i}^{T} P_{i}+P_{i} A_{i}+R_{i, 1}+Q_{i} \\
& +\frac{d}{2} A_{i}^{T}\left(Z_{i, 1}+Z_{i, 2}\right) A_{i}-\frac{2}{d} e^{\alpha d / 2}-\alpha P_{i}, \\
& Z_{i}=Z_{i, 1}+Z_{i, 2}, \quad R_{i}=R_{i, 1}+R_{i, 2}, \quad P_{i} \leq \beta P_{j}, \\
& R_{i, 1} \leq \beta R_{j, 1}, \quad R_{i, 2} \leq \beta R_{j, 2}, \quad Q_{i} \leq \beta Q_{j} \\
& Z_{i, 1} \leq \beta Z_{j, 1}, \quad Z_{i, 2} \leq \beta Z_{j, 2}, \quad i, j \in[1,2, \ldots, m], \\
& \lambda_{1}=\max _{i \in M}\left\{\lambda_{\max }\left(P_{i}\right)\right\}, \quad \lambda_{2}=\max _{i \in M}\left\{\lambda_{\max }\left(R_{i, 1}\right)\right\}, \\
& \lambda_{3}=\max _{i \in M}\left\{\lambda_{\max }\left(R_{i, 2}\right)\right\}, \quad \lambda_{4}=\max _{i \in M}\left\{\lambda_{\max }\left(Q_{i}\right)\right\}, \\
& \lambda_{5}=\max _{i \in M}\left\{\lambda_{\max }\left(Z_{i, 1}\right)\right\}, \quad \lambda_{6}=\max _{i \in M}\left\{\lambda_{\max }\left(Z_{i, 2}\right)\right\} \text {, } \\
& \lambda_{7}=\lambda_{\max }(H), \quad \lambda_{8}=\min _{i \in M}\left\{\lambda_{\min }\left(P_{i}\right)\right\}, \\
& \eta_{1}=\lambda_{1}+\frac{d}{2} e^{\alpha d / 2} \lambda_{2}+\frac{d}{2} e^{\alpha d} \lambda_{3}+h_{\max } e^{\alpha h_{\max }} \lambda_{4}, \\
& \eta_{2}=\frac{d^{2}}{4} \rho^{2} \lambda_{5} e^{\alpha d / 2}+\frac{d^{2}}{2} \rho^{2} \lambda_{6} e^{\alpha d}+\frac{d}{2} \lambda_{7} \gamma \\
& C_{1}=\sup _{-d \leq t_{0} \leq 0}\left\{x^{T}\left(\bar{t}_{0}\right) x\left(\bar{t}_{0}\right)\right\}, \\
& C_{2}=\left(\beta^{N} e^{\alpha T_{f}} \eta_{1} C_{1}+\beta^{N} e^{\alpha T_{f}}\right. \\
& \times\left(\frac{d^{2}}{4} \rho^{2} \lambda_{5} e^{\alpha d / 2}+\frac{d^{2}}{2} \rho^{2} \lambda_{6} e^{\alpha d}\right. \\
& \left.\left.+\frac{d}{2} e^{\alpha T_{f}} \lambda_{7} \gamma\right)\right)\left(\lambda_{8}\right)^{-1} .
\end{aligned}
$$

The left of inequality (6) is a symmetric matrix. Thus, the symmetric terms are denoted by “*”. $\lambda_{\max }\left(P_{i}\right)$ represents the maximum eigenvalue of $P_{i}$.

Proof. Construct the multiply Lyapunov function as follows:

$$
\begin{gathered}
V(t)=V_{i}(t)=V_{i, 1}(t)+V_{i, 2}(t)+V_{i, 3}(t)+V_{i, 4}(t), \\
V_{i, 1}(t)=x^{T}(t) P_{i} x(t), \\
V_{i, 2}(t)=\int_{t-(d / 2)}^{t} x^{T}(s) e^{-\alpha(s-t)} R_{i, 1} x(s) d s \\
+\int_{t-d}^{t-(d / 2)} x^{T}(s) e^{-\alpha(s-t)} R_{i, 2} x(s) d s,
\end{gathered}
$$

$$
\begin{gathered}
V_{i, 3}(t)=\int_{t-h(t)}^{t} x^{T}(s) e^{-\alpha(s-t)} Q_{i} x(s) d s, \\
V_{i, 4}(t)=\int_{-d / 2}^{0} \int_{t+\theta}^{t} \dot{x}^{T}(s) e^{-\alpha(s-t)} Z_{i, 1} \dot{x}(s) d s d \theta \\
+\int_{-d}^{-d / 2} \int_{t+\theta}^{t} \dot{x}^{T}(s) e^{-\alpha(s-t)} Z_{i, 2} \dot{x}(s) d s d \theta .
\end{gathered}
$$

Calculate the derivatives of $V_{i, 1}(t), V_{i, 2}(t), V_{i, 3}(t)$, and $V_{i, 4}(t)$ as

$$
\begin{aligned}
\dot{V}_{i, 1}(t)= & x^{T}(t)\left[A_{i}^{T} P_{i}+P_{i} A_{i}\right] x(t) \\
& +x^{T}(t-h(t)) B_{i}^{T} P_{i} x(t)+w^{T}(t) G_{i}^{T} P_{i} x(t) \\
& +x^{T}(t) P_{i} B_{i} x(t-h(t))+x^{T}(t) P_{i} G_{i} w(t)
\end{aligned}
$$

Furthermore, it follows that

$$
\begin{aligned}
\dot{V}_{i, 1}(t)-\alpha V_{i, 1}= & x^{T}(t)\left[A_{i}^{T} P_{i}+P_{i} A_{i}\right] x(t) \\
& +x^{T}(t-h(t)) B_{i}^{T} P_{i} x(t)+w^{T}(t) G_{i}^{T} P_{i} x(t) \\
& +x^{T}(t) P_{i} B_{i} x(t-h(t))+x^{T}(t) P_{i} G_{i} w(t) \\
& -\alpha x^{T}(t) P_{i} x(t) \\
\dot{V}_{i, 2}(t)= & \alpha V_{i, 2}(t)+x^{T}(t) R_{i, 1} x(t) \\
& +x^{T}\left(t-\frac{d}{2}\right) e^{\alpha d / 2}\left[R_{i, 2}-R_{i, 1}\right] x\left(t-\frac{d}{2}\right) \\
& -x^{T}(t-d) e^{\alpha d} R_{i, 2} x(t-d) \\
\dot{V}_{i, 3}(t)= & \alpha V_{i, 3}(t)+x^{T}(t) Q_{i} x(t) \\
& -x^{T}(t-h(t))(1-\dot{h}(t)) \\
& \times e^{\alpha h(t)} Q_{i} x(t-h(t)) \\
\leq & \alpha V_{i, 3}(t)+x^{T}(t) Q_{i} x(t) \\
& -x^{T}(t-h(t))(1-k) e^{\alpha h(t)} Q_{i} x(t-h(t)) \\
\leq & \alpha V_{i, 3}(t)+x^{T}(t) Q_{i} x(t) \\
& -h(t))(1-k) Q_{i} x(t-h(t))
\end{aligned}
$$$$
\dot{V}_{i, 4}(t)=\alpha V_{i, 4}(t)+\frac{d}{2} \dot{x}^{T}(t)\left[Z_{i, 1}+Z_{i, 2}\right] \dot{x}(t)
$$$$
-\int_{t-(d / 2)}^{t} \dot{x}^{T}(s) e^{-\alpha(s-t)} Z_{i, 1} \dot{x}(s) d s
$$ 


$$
\begin{gathered}
-\int_{t-d}^{t-(d / 2)} x^{T}(s) e^{-\alpha(s-t)} Z_{i, 2} x(s) d s \\
=\alpha V_{i, 4}(t)+\frac{d}{2}\left[A_{i} x(t)+B_{i} x\right. \\
\left.\times(t-h(t))+G_{i} w(t)\right]^{T} \\
\times\left[Z_{i, 1}+Z_{i, 2}\right] \\
\times\left[A_{i} x(t)+B_{i} x(t-h(t))+G_{i} w(t)\right] \\
-\int_{t-(d / 2)}^{t} \dot{x}^{T}(s) e^{-\alpha(s-t)} Z_{i, 1} \dot{x}(s) d s \\
-\int_{t-d}^{t-(d / 2)} x^{T}(s) e^{-\alpha(s-t)} Z_{i, 2} x(s) d s .
\end{gathered}
$$

Due to the Jensen inequality, inequality (15) holds

$$
\begin{aligned}
\int_{t-(d / 2)}^{t} & \dot{x}^{T}(s) e^{-\alpha(s-t)} Z_{i, 1} \dot{x}(s) d s \\
\geq & \frac{2}{d}\left[x(t)-x\left(t-\frac{d}{2}\right)\right]^{T} \\
& \times e^{\alpha d / 2} Z_{i, 1}\left[x(t)-x\left(t-\frac{d}{2}\right)\right], \\
\int_{t-d}^{t-(d / 2)} & x^{T}(s) e^{-\alpha(s-t)} Z_{i, 2} x(s) d s
\end{aligned}
$$$$
\dot{V}_{i}(t)-\alpha V_{i}(t) \leq\left[\begin{array}{c}
x(t) \\
x(t-h(t)) \\
x\left(t-\frac{d}{2}\right) \\
x(t-d) \\
w(t)
\end{array}\right]^{T}
$$$$
\times\left[\begin{array}{ccccc}
\xi_{11} & P_{i} B_{i}+\frac{d}{2} A_{i}^{T} Z_{i} B_{i} & \frac{2}{d} e^{\alpha d / 2} Z_{i, 1} & 0 & P_{i} G_{i}+\frac{d}{2} A_{i}^{T} Z_{i} G_{i} \\
* & \frac{d}{2} B_{i}^{T} Z_{i} B_{i}-(1-k) Q & 0 & 0 & \frac{d}{2} B_{i}^{T} Z_{i} G_{i} \\
* & * & e^{\alpha d / 2} R_{i}-\frac{2}{d} e^{\alpha d / 2} Z_{i} & \frac{2}{d} e^{\alpha d} Z_{i, 2} & 0 \\
* & * & * & -e^{\alpha d} R_{i, 2}-\frac{2}{d} e^{\alpha d} Z_{i, 2} & 0 \\
* & * & * & * & \frac{d}{2} G_{i}^{T} Z_{i} G_{i}
\end{array}\right]
$$$$
\times\left[\begin{array}{c}
x(t) \\
x(t-h(t)) \\
x\left(t-\frac{d}{2}\right) \\
x(t-d) \\
w(t)
\end{array}\right]
$$

By (14) and (15), we obtain

$$
\begin{aligned}
\dot{V}_{i, 4}(t) \leq & \alpha V_{i, 4}(t)+\frac{d}{2}\left[A_{i} x(t)+B_{i} x(t-h(t))+G_{i} w(t)\right]^{T} \\
& \times\left[Z_{i, 1}+Z_{i, 2}\right]\left[A_{i} x(t)+B_{i} x(t-h(t))+G_{i} w(t)\right] \\
& -\frac{2}{d}\left[x(t)-x\left(t-\frac{d}{2}\right)\right]^{T} \\
& \times e^{\alpha d / 2} Z_{i, 1}\left[x(t)-x\left(t-\frac{d}{2}\right)\right] \\
& -\frac{2}{d}\left[x\left(t-\frac{d}{2}\right)-x(t-d)\right]^{T} \\
& \times e^{\alpha d} Z_{i, 2}\left[x\left(t-\frac{d}{2}\right)-x(t-d)\right] .
\end{aligned}
$$

From (11), (12), (13), and (16), it is easy to get 
According to the definition of finite-time boundedness, the rest of the proof will be divided into two steps. Under the given conditions, we need to prove that $x^{T}(t) x(t)<c_{2}$ and $c_{1}<c_{2}$, respectively.

(i) We will prove that $x^{T}(t) x(t)<C_{2}$ holds for all $t$ on $\left[0, T_{f}\right]$.

By (6) and (17), inequality (18) holds

$$
\dot{V}_{i}(t)-\alpha V_{i}(t)<\frac{d}{2} w^{T}(t) H w(t) .
$$

Since $(d / d t)\left(e^{-\alpha t} V_{i}(t)\right)=e^{-\alpha t}\left[\dot{V}_{i}(t)-\alpha V_{i}(t)\right]$, inequality (18) can be transformed into

$$
\frac{d}{d t}\left(e^{-\alpha t} V_{i}(t)\right)<\frac{d}{2} e^{-\alpha t} w^{T}(t) H w(t)
$$

Let $t_{k}$ stand for the instant of the Kth switching.

Integrating from $t_{k}$ to $t$ on both sides of (19), it follows that

$$
V_{i}(t)<e^{\alpha\left(t-t_{k}\right)} V_{i}\left(t_{k}\right)+\frac{d}{2} \int_{t_{k}}^{t} w^{T}(s) e^{\alpha(t-s)} H w(s) d s .
$$

Notice that $P_{i} \leq \beta P_{j}, R_{i, 1} \leq \beta R_{j, 1}, R_{i, 2} \leq \beta R_{j, 2}, Q_{i} \leq \beta Q_{j}$, $Z_{i, 1} \leq \beta Z_{j, 1}, Z_{i, 2} \leq \beta Z_{j, 2}, i$, and $j \in[1,2, \ldots, m]$ and the continuity of $x(t)$, hence (21) holds

$$
V_{i}(t)<\beta e^{\alpha\left(t-t_{k}\right)} V_{i}\left(t_{k^{-}}\right)+\frac{d}{2} \int_{t_{k}}^{t} w^{T}(s) e^{\alpha(t-s)} H w(s) d s,
$$

where $t_{k^{-}}$denotes the instant just before $t_{k}$

It is easy to see

$$
V_{i}\left(t_{k^{-}}\right)<e^{\alpha\left(t_{k}-t_{k-1}\right)} V_{i}\left(t_{k-1}\right)+\frac{d}{2} \int_{t_{k-1}}^{t_{k}} w^{T}(s) e^{\alpha(t-s)} H w(s) d s
$$

Then (23) is obtained

$$
\begin{aligned}
V_{i}(t)< & \beta^{2} e^{\alpha\left(t-t_{k-1}\right)} V_{i}\left(t_{(k-1)^{-}}\right) \\
& +\frac{d}{2} \beta e^{\alpha\left(t-t_{k}\right)} \int_{t_{k-1}}^{t_{k}} w^{T}(s) e^{\alpha(t-s)} H w(s) d s \\
& +\frac{d}{2} \int_{t_{k}}^{t} w^{T}(s) e^{\alpha(t-s)} H w(s) d s .
\end{aligned}
$$

Assume the switching number of $\sigma(t)$ over $\left[0, T_{f}\right]$ is $N$. (24) is obtained via the iterative calculation

$$
\begin{aligned}
V_{i}(t)< & \beta^{N} e^{\alpha t} V_{i}(0)+\frac{d}{2} \beta^{N} e^{\alpha\left(t-t_{1}\right)} \\
& \times \int_{t_{0}}^{t_{1}} w^{T}(s) e^{\alpha(t-s)} H w(s) d s+\cdots \\
& +\frac{d}{2} \beta e^{\alpha\left(t-t_{k}\right)} \int_{t_{k-1}}^{t_{k}} w^{T}(s) e^{\alpha(t-s)} H w(s) d s \\
& +\frac{d}{2} \int_{t_{k}}^{T_{f}} w^{T}(s) e^{\alpha(t-s)} H w(s) d s \\
e^{\alpha T_{f}}> & e^{\alpha\left(T_{f}-t_{1}\right)}>e^{\alpha\left(T_{f}-t_{2}\right)}>\cdots>e^{\alpha\left(T_{f}-t_{k}\right)}>1
\end{aligned}
$$$$
\text { for } t \in\left[0, T_{f}\right], \beta^{N} \geq \beta^{N-1} \geq \cdots \geq \beta \geq 1 \text {. }
$$

$$
\begin{gathered}
V_{i}(t)<\beta^{N} e^{\alpha T_{f}} V_{i}(0)+\frac{d}{2} \beta^{N} e^{\alpha T_{f}} \\
\quad \times \int_{t_{0}}^{t_{1}} w^{T}(s) e^{\alpha(t-s)} H w(s) d s+\cdots \\
+\frac{d}{2} \beta^{N} e^{\alpha T_{f}} \int_{t_{k-1}}^{t_{k}} w^{T}(s) e^{\alpha(t-s)} H w(s) d s \\
+\frac{d}{2} e^{\alpha T_{f}} \beta^{N} \int_{t_{k}}^{t} w^{T}(s) e^{\alpha(t-s)} H w(s) d s \\
V_{i}(t)<\beta^{N} e^{\alpha T_{f}} V_{i}(0)+\frac{d}{2} e^{\alpha T_{f}} \beta^{N} \int_{0}^{t} w^{T}(s) e^{\alpha(t-s)} H w(s) d s .
\end{gathered}
$$

On the other hand, since $1 \leq e^{\alpha(t-s)} \leq e^{\alpha t} \leq e^{\alpha T_{f}}$ and $H \leq \lambda_{\text {max }}(H)$, we have

$$
\begin{aligned}
\frac{d}{2} e^{\alpha T_{f}} & \beta^{N} \int_{0}^{t} w^{T}(s) e^{\alpha(t-s)} H w(s) d s \\
& \leq \frac{d}{2} e^{2 \alpha T_{f}} \beta^{N} \lambda_{\max }(H) \int_{0}^{t} w^{T}(s) w(s) d s \\
& \leq \frac{d}{2} e^{2 \alpha t} \beta^{N} \lambda_{\max }(H) \gamma .
\end{aligned}
$$

Applying the above inequality to (27), we get

$$
V_{i}(t)<\beta^{N} e^{\alpha T_{f}} V_{i}(0)+\frac{d}{2} e^{2 \alpha T_{f}} \beta^{N} \lambda_{\max }(H) \gamma
$$


With respect to $V_{i}(0)$ in (29), it is processed as follows:

$$
\begin{aligned}
& V_{i}(0)=x^{T}(0) P_{i} x(0)+\int_{-d / 2}^{0} x^{T}(s) e^{-\alpha s} R_{i, 1} x(s) d s \\
& +\int_{-d}^{-d / 2} x^{T}(s) e^{-\alpha s} R_{i, 2} x(s) d s \\
& +\int_{-h(0)}^{0} x^{T}(s) e^{-\alpha s} Q_{i} x(s) d s \\
& +\int_{-d / 2}^{0} \int_{\theta}^{0} \dot{x}^{T}(s) e^{-\alpha s} Z_{i, 1} \dot{x}(s) d s d \theta \\
& +\int_{-d}^{-d / 2} \int_{\theta}^{0} \dot{x}^{T}(s) e^{-\alpha s} Z_{i, 2} \dot{x}(s) d s d \theta \\
& <\lambda_{\text {max }}\left(P_{i}\right) \sup _{-d \leq t \leq 0}\left\{x^{T}\left(\bar{t}_{0}\right) x\left(\bar{t}_{0}\right)\right\} \\
& +\frac{d}{2} e^{\alpha d / 2} \lambda_{\max }\left(R_{i, 1}\right) \sup _{-d \leq t \leq 0}\left\{x^{T}\left(\bar{t}_{0}\right) x\left(\bar{t}_{0}\right)\right\} \\
& +\frac{d}{2} e^{\alpha d} \lambda_{\max }\left(R_{i, 2}\right) \sup _{-d \leq t \leq 0}\left\{x^{T}\left(\bar{t}_{0}\right) x\left(\bar{t}_{0}\right)\right\} \\
& +h_{\max } e^{\alpha h_{\max }} \lambda_{\max }\left(Q_{i}\right) \sup _{-d \leq t \leq 0}\left\{x^{T}\left(\bar{t}_{0}\right) x\left(\bar{t}_{0}\right)\right\} \\
& +\int_{-d / 2}^{0}-\theta \rho^{2} \lambda_{\max }\left(Z_{i, 1}\right) e^{-\alpha \theta} d \theta \\
& +\int_{-d}^{-d / 2}-\theta \rho^{2} \lambda_{\max }\left(Z_{i, 2}\right) e^{-\alpha \theta} d \theta \\
& <\lambda_{\max }\left(P_{i}\right) \sup _{-d \leq t \leq 0}\left\{x^{T}\left(\bar{t}_{0}\right) x\left(\bar{t}_{0}\right)\right\} \\
& +\frac{d}{2} e^{\alpha d / 2} \lambda_{\max }\left(R_{i, 1}\right) \sup _{-d \leq t \leq 0}\left\{x^{T}\left(\bar{t}_{0}\right) x\left(\bar{t}_{0}\right)\right\} \\
& +\frac{d}{2} e^{\alpha d} \lambda_{\max }\left(R_{i, 2}\right) \sup _{-d \leq t \leq 0}\left\{x^{T}\left(\bar{t}_{0}\right) x\left(\bar{t}_{0}\right)\right\} \\
& +h_{\max } e^{\alpha h_{\max }} \lambda_{\max }\left(Q_{i}\right) \sup _{-d \leq t \leq 0}\left\{x^{T}\left(\bar{t}_{0} t\right) x\left(\bar{t}_{0}\right)\right\} \\
& +\frac{d}{2} \cdot \frac{d}{2} \rho^{2} \lambda_{\max }\left(Z_{i, 1}\right) e^{\alpha d / 2}+\frac{d}{2} \cdot d \rho^{2} \lambda_{\max }\left(Z_{i, 2}\right) e^{\alpha d} .
\end{aligned}
$$

Applying known mathematical relationships to (30), (31) can be obtained as

$$
\begin{aligned}
V_{i}(0)< & \lambda_{1} C_{1}+\frac{d}{2} e^{\alpha d / 2} \lambda_{2} C_{1}+\frac{d}{2} e^{\alpha d} \lambda_{3} C_{1} \\
& +h_{\max } e^{\alpha h_{\max }} \lambda_{4} C_{1}+\frac{d^{2}}{4} \rho^{2} \lambda_{5} e^{\alpha d / 2}+\frac{d^{2}}{2} \rho^{2} \lambda_{6} e^{\alpha d}
\end{aligned}
$$

Inequality (32) is obtained via (29) and (31) as

$$
\begin{aligned}
V_{i}(t)< & \beta^{N} e^{\alpha T_{f}} \\
& \times\left(\lambda_{1}+\frac{d}{2} e^{\alpha d / 2} \lambda_{2}+\frac{d}{2} e^{\alpha d} \lambda_{3}+h_{\max } e^{\alpha h_{\max }} \lambda_{4}\right) C_{1} \\
& +\beta^{N} e^{\alpha T_{f}}\left(\frac{d^{2}}{4} \rho^{2} \lambda_{5} e^{\alpha d / 2}+\frac{d^{2}}{2} \rho^{2} \lambda_{6} e^{\alpha d}\right. \\
& \left.\quad+\frac{d}{2} e^{\alpha T_{f}} \lambda_{7} \gamma\right) \\
= & \beta^{N} e^{\alpha T_{f}} \eta_{1} C_{1}+\beta^{N} e^{\alpha T_{f}} \\
& \times\left(\frac{d^{2}}{4} \rho^{2} \lambda_{5} e^{\alpha d / 2}+\frac{d^{2}}{2} \rho^{2} \lambda_{6} e^{\alpha d}+\frac{d}{2} e^{\alpha T_{f}} \lambda_{7} \gamma\right)
\end{aligned}
$$

According to the definition of $V_{i}(t)$, inequality (33) holds

$$
\begin{aligned}
V_{i}(t)>x^{T}(t) P_{i} x(t) & \geq \lambda_{\min }\left(P_{i}\right) x^{T}(t) x(t) \\
& \geq \min _{i \in M}\left\{\lambda_{\min }\left(P_{i}\right)\right\} x^{T}(t) x(t) .
\end{aligned}
$$

Then the following holds based on (32) and (33):

$$
\begin{aligned}
x^{T}(t) x(t)< & \left(\beta^{N} e^{\alpha T_{f}} \eta_{1} C_{1}+\beta^{N} e^{\alpha T_{f}}\right. \\
& \times\left(\frac{d^{2}}{4} \rho^{2} \lambda_{5} e^{\alpha d / 2}+\frac{d^{2}}{2} \rho^{2} \lambda_{6} e^{\alpha d}\right. \\
& \left.\left.+\frac{d}{2} e^{\alpha T_{f}} \lambda_{7} \gamma\right)\right)\left(\lambda_{8}\right)^{-1} \\
& =C_{2} .
\end{aligned}
$$

(ii) Next, $C_{1}<C_{2}$ will be demonstrated. By (7), we have

$$
\begin{gathered}
\frac{T_{f}}{\tau_{a}}>\frac{\ln C_{1}+\ln \lambda_{8}-\ln \left(\eta_{1} C_{1}+\eta_{2}\right)-\alpha T_{f}}{\ln \beta}, \\
N>\frac{T_{f}}{\tau_{a}}>\frac{\ln C_{1}+\ln \lambda_{8}-\ln \left(\eta_{1} C_{1}+\eta_{2}\right)-\alpha T_{f}}{\ln \beta}, \\
\ln \left(\eta_{1} C_{1}+\eta_{2}\right)-\ln \lambda_{8}>\ln C_{1}-N \ln \beta-\alpha T_{f}, \\
\frac{\eta_{1} C_{1}+\eta_{2}}{\lambda_{8}} e^{\alpha T_{f}} \beta^{N}>C_{1} .
\end{gathered}
$$


On the other hand, due to $e^{\alpha T_{f}} \geq 1$, there exist the following mathematical relations:

$$
\begin{array}{r}
C_{2}=\left(\beta^{N} e^{\alpha T_{f}} \eta_{1} C_{1}+\beta^{N} e^{\alpha T_{f}}\right. \\
\times\left(\frac{d^{2}}{4} \rho^{2} \lambda_{5} e^{\alpha d / 2}+\frac{d^{2}}{2} \rho^{2} \lambda_{6} e^{\alpha d}\right. \\
\left.\left.+\frac{d}{2} e^{\alpha T_{f}} \lambda_{7} \gamma\right)\right)\left(\lambda_{8}\right)^{-1} \\
\geq\left(\beta^{N} e^{\alpha T_{f}} \eta_{1} C_{1}+\beta^{N} e^{\alpha T_{f}}\right. \\
\times\left(\frac{d^{2}}{4} \rho^{2} \lambda_{5} e^{\alpha d / 2}+\frac{d^{2}}{2} \rho^{2} \lambda_{6} e^{\alpha d}\right. \\
\left.\left.+\frac{d}{2} \lambda_{7} \gamma\right)\right)\left(\lambda_{8}\right)^{-1} \\
=\frac{\eta_{1} C_{1}+\eta_{2}}{\lambda_{8}} e^{\alpha T_{f}} \beta^{N} .
\end{array}
$$

Combining (38) and (39), we get $c_{2}>c_{1}$.

By (i) and (ii), the system (1) satisfies the definition of finite-time boundedness under given conditions. This completes the proof of Theorem 8 .

Remark 9. Notice that (6) is not a linear matrix inequality. Thus, it cannot be directly solved via LMI toolbox. Before solving (6), the inequality can be transformed to a linear matrix inequality by specifying the value of $\alpha$.

\section{A Numerical Example}

An example is presented to illustrate Theorem 8. Consider

$$
\begin{aligned}
& \dot{x}(t)=A_{\sigma(t)} x(t)+B_{\sigma(t)} x(t-h(t))+G_{\sigma(t)} w(t), \quad t \geq 0, \\
& x(t)=\varphi(t), \quad t \in[-d, 0), \\
& A_{1}=\left[\begin{array}{ccc}
-1.7 & 1.7 & 0 \\
1.3 & -1 & 0.7 \\
0.7 & 1 & -0.6
\end{array}\right], \quad A_{2}=\left[\begin{array}{ccc}
1 & -1 & 0 \\
0.7 & 0 & -0.6 \\
1.7 & 0 & -1.7
\end{array}\right] \text {, } \\
& B_{1}=\left[\begin{array}{ccc}
1.5 & -1.7 & 0.1 \\
-1.3 & 1 & -0.3 \\
-0.7 & 1 & 0.6
\end{array}\right], \quad B_{2}=\left[\begin{array}{ccc}
-1 & -0.3 & 0.1 \\
1.3 & -0.1 & 0.6 \\
1.5 & 0.1 & 1.8
\end{array}\right] \text {, } \\
& G_{1}=G_{2}=\left[\begin{array}{lll}
1 & 0 & 0 \\
0 & 1 & 0 \\
0 & 0 & 1
\end{array}\right] \\
& w(t)=\left[\begin{array}{c}
0.03 \sin (t) \\
0.02 \cos (2 t) \\
0.015(\sin (t+1)+\cos (t-2))
\end{array}\right] \text {, }
\end{aligned}
$$

$$
\begin{gathered}
h(t)=0.5 t, \quad d=0.2 \\
\varphi(t) \equiv\left[\begin{array}{lll}
0.5 & 0.1 & 0
\end{array}\right]^{T}, \quad \forall t \in[-0.2,0] \\
\max |\dot{\varphi}(t)| \leq \rho=0, \quad \dot{h}(t) \leq k=0.5, \quad C_{1}=0.26 .
\end{gathered}
$$

Let $\alpha=0.02, \beta=1.1$, and $T_{f}=10$, then $h(t) \leq h_{\max }=$ $0.5 * 10=5$ and $\int_{0}^{T_{f}} w^{T}(s) w(s) d t \leq \gamma \approx 0.022$.

Solving (6) leads to feasible solutions that

$$
\begin{aligned}
& P_{1}=\left[\begin{array}{ccc}
0.8983 & -0.0167 & 0.1555 \\
-0.0167 & 1.0898 & -0.3930 \\
0.1555 & -0.3930 & 0.9754
\end{array}\right] \\
& P_{2}=\left[\begin{array}{ccc}
0.6101 & 0.1828 & -0.1480 \\
0.1828 & 0.8908 & -0.3026 \\
-0.1480 & -0.3026 & 0.8153
\end{array}\right] \text {, } \\
& R_{1,1}=\left[\begin{array}{ccc}
0.7188 & -0.1052 & 0.0283 \\
-0.1052 & 0.7458 & -0.1300 \\
0.0283 & -0.1300 & 0.7114
\end{array}\right] \text {, } \\
& R_{1,2}=\left[\begin{array}{ccc}
1.3854 & -0.1336 & 0.0209 \\
-0.1336 & 1.3613 & -0.1532 \\
0.0209 & -0.1532 & 1.3368
\end{array}\right] \text {, } \\
& R_{2,1}=\left[\begin{array}{ccc}
0.5289 & 0.0089 & -0.0566 \\
0.0089 & 0.6200 & -0.0083 \\
-0.0566 & -0.0083 & 0.6743
\end{array}\right] \text {, } \\
& R_{2,2}=\left[\begin{array}{ccc}
1.1615 & 0.0282 & -0.0465 \\
0.0282 & 1.2555 & -0.0175 \\
-0.0465 & -0.0175 & 1.3518
\end{array}\right] \text {, } \\
& Q_{1}=\left[\begin{array}{ccc}
4.2184 & -0.5908 & -0.1106 \\
-0.5908 & 4.4575 & -0.3066 \\
-0.1106 & -0.3066 & 4.0548
\end{array}\right] \text {, } \\
& Q_{2}=\left[\begin{array}{lll}
3.8150 & 0.0518 & 0.0675 \\
0.0518 & 3.7399 & 0.0356 \\
0.0675 & 0.0356 & 4.2709
\end{array}\right], \\
& Z_{1,1}=\left[\begin{array}{ccc}
0.3150 & -0.0492 & -0.0003 \\
-0.0492 & 0.2950 & -0.0639 \\
-0.0003 & -0.0639 & 0.3082
\end{array}\right] \text {, } \\
& Z_{1,2}=\left[\begin{array}{lll}
0.4060 & 0.0002 & 0.0006 \\
0.0002 & 0.4036 & 0.0013 \\
0.0006 & 0.0013 & 0.3934
\end{array}\right] \text {, } \\
& Z_{2,1}=\left[\begin{array}{ccc}
0.2124 & 0.0176 & -0.0190 \\
0.0176 & 0.2812 & -0.0034 \\
-0.0190 & -0.0034 & 0.3118
\end{array}\right] \text {, } \\
& Z_{2,2}=\left[\begin{array}{ccc}
0.3934 & 0.0070 & 0.0039 \\
0.0070 & 0.3920 & -0.0131 \\
0.0039 & -0.0131 & 0.3961
\end{array}\right] \text {, }
\end{aligned}
$$




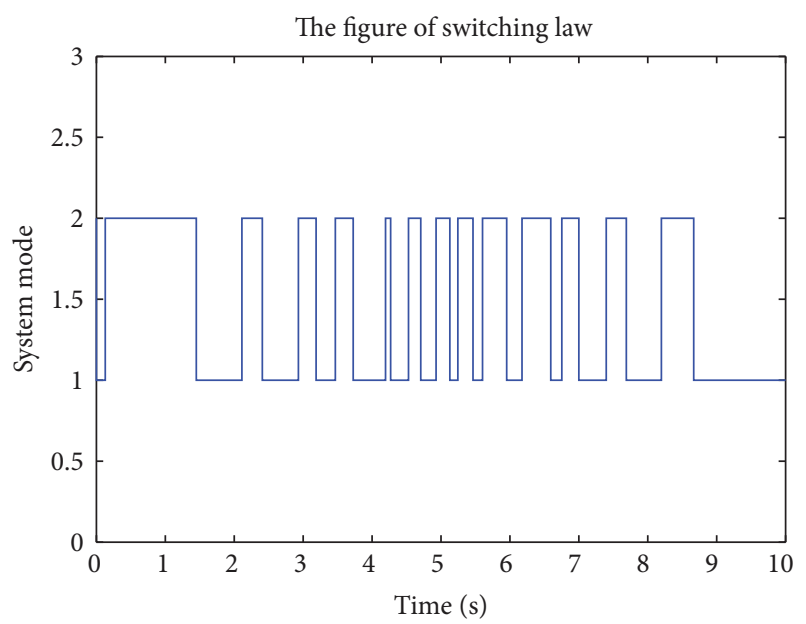

FIgURE 1: The diagram of switching law.

$$
\begin{gathered}
H=\left[\begin{array}{ccc}
12.5148 & 0.6115 & 0.1387 \\
0.6115 & 13.0302 & -0.5248 \\
0.1387 & -0.5248 & 12.4327
\end{array}\right], \\
\lambda_{1}=1.4539, \quad \lambda_{2}=0.9114, \quad \lambda_{3}=1.5749, \\
\lambda_{4}=4.9735, \quad \lambda_{5}=0.2449, \quad \lambda_{6}=0.1192, \\
\lambda_{7}=13.5367, \quad \lambda_{8}=0.5200 .
\end{gathered}
$$

Further, we get that $C_{2}=2000.6421>C_{1}$ and $\tau_{a}<1.8263$. The simulation of the numerical example is performed and its results are shown in Figures 1 and 2. From Figure 1, one can get that $\tau_{a}<1.8263$ holds. From Figure 2, it is easily found that the value of $x^{T}(t) x(t)$ remains within $C_{2}$ for $t \in\left[0, T_{f}\right]$. So, the system is indeed finite-time bounded over $\left[0, T_{f}\right]$.

\section{Conclusion}

(1) For the switched linear system, a new definition on finite-time boundedness is proposed which can reduce some complex matrix calculations.

(2) Under given conditions, the sufficient conditions which guarantee the system is finite-time bounded are given for the switched linear system with time-varying delay and external disturbance.

(3) In the future study, a challenging research topic is how to ensure the switched system with time-varying delay remains finite-time bounded for any switching signal.

\section{Conflict of Interests}

The authors (Yanke Zhong and Tefang Chen) declare that there is no conflict of interests regarding the publication of this paper.

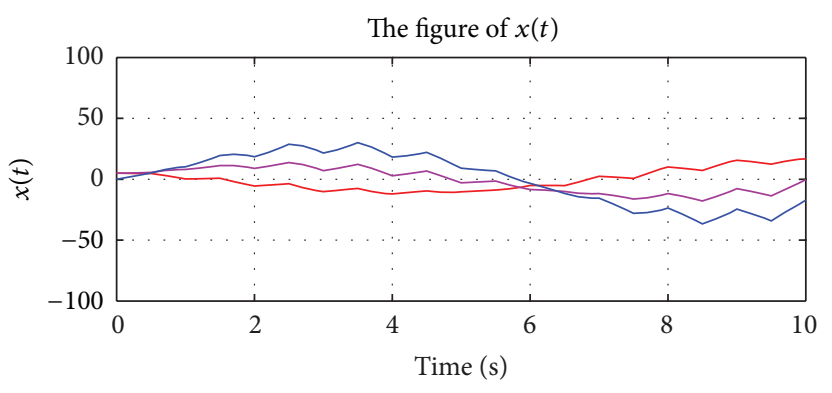

(a)

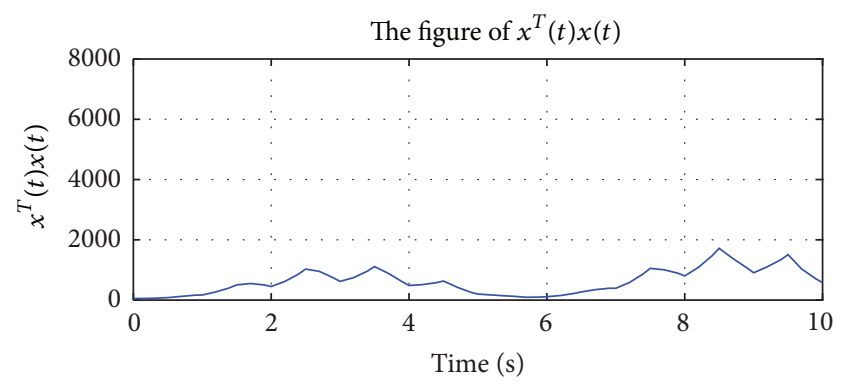

(b)

FIgURE 2: The diagrams of $x(t)$ and $x^{T}(t) x(t)$.

\section{Acknowledgment}

This work was supported by the National Natural Science Foundation of China under Grant no. 61273158.

\section{References}

[1] Y. Sun, "Delay-independent stability of switched linear systems with unbounded time-varying delays," Abstract and Applied Analysis, vol. 2012, Article ID 560897, 11 pages, 2012.

[2] D. Liberzon, Switching in Systems and Control, Birkhäuser, Boston, Mass, USA, 2003.

[3] H. F. Sun, J. Zhao, and X. D. Gao, "Stability of switched linear systems with delayed perturbation," Control and Decision, vol. 17, no. 4, pp. 431-434, 2002.

[4] Z. Li, Y. Soh, and C. Wen, Switched and Impulsive Systems: Analysis, Design, and Applications, vol. 313, Springer, Berlin, Germany, 2005.

[5] R. Goebel, R. G. Sanfelice, and A. R. Teel, "Hybrid dynamical systems: robust stability and control for systems that combine continuous-time and discrete-time dynamics," IEEE Control Systems Magazine, vol. 29, no. 2, pp. 28-93, 2009.

[6] M. Margaliot and J. P. Hespanha, "Root-mean-square gains of switched linear systems: a variational approach," Automatica, vol. 44, no. 9, pp. 2398-2402, 2008.

[7] R. Shorten, F. Wirth, O. Mason, K. Wulff, and C. King, "Stability criteria for switched and hybrid systems," SIAM Review, vol. 49, no. 4, pp. 545-592, 2007.

[8] J.-W. Lee and P. P. Khargonekar, "Optimal output regulation for discrete-time switched and Markovian jump linear systems," SIAM Journal on Control and Optimization, vol. 47, no. 1, pp. 4072, 2008.

[9] M. S. Branicky, V. S. Borkar, and S. K. Mitter, "A unified framework for hybrid control: model and optimal control 
theory," IEEE Transactions on Automatic Control, vol. 43, no. 1, pp. 31-45, 1998.

[10] J. N. Lu and G. Y. Zhao, "Stability analysis based on LMI for switched systems with time delay," Journal of Southern Yangtze University, vol. 5, no. 2, pp. 171-173, 2006.

[11] L. Y. Zhao and Z. Q. Zhang, "Stability analysis of a class of switched systems with time delay," Control and Decision, vol. 26, no. 7, pp. 1113-1116, 2011.

[12] J. Lian, C. Mu, and P. Shi, "Asynchronous H-infinity Filtering for switched stochastic systems with time-varying delay," Information Sciences, pp. 200-212, 2013.

[13] Y. Sun, "Stabilization of switched systems with nonlinear impulse effects and disturbances," IEEE Transactions on Automatic Control, vol. 56, no. 11, pp. 2739-2743, 2011.

[14] X. Lin, H. Du, and S. Li, "Finite-time boundedness and L2gain analysis for switched delay systems with norm-bounded disturbance," Applied Mathematics and Computation, pp. 59825993, 2011.

[15] J. P. Hespanha and A. S. Morse, "Stability of switched systems with average dwell-time," in Proceedings of the IEEE Conference on Decision and Control, pp. 2655-2660, 1999. 


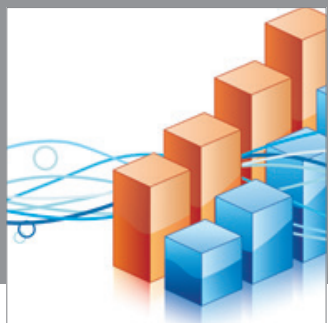

Advances in

Operations Research

mansans

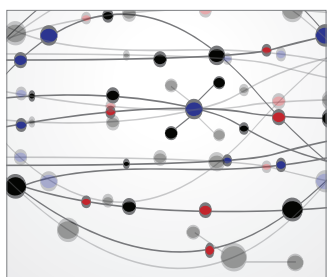

The Scientific World Journal
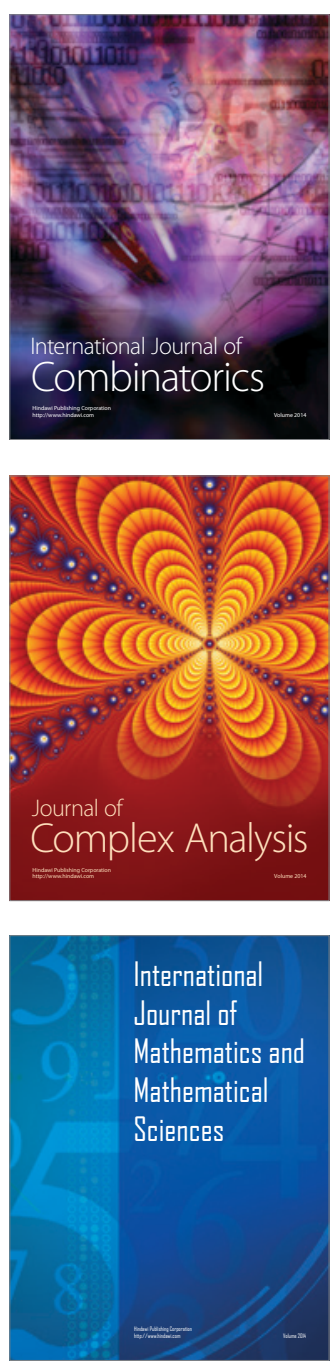
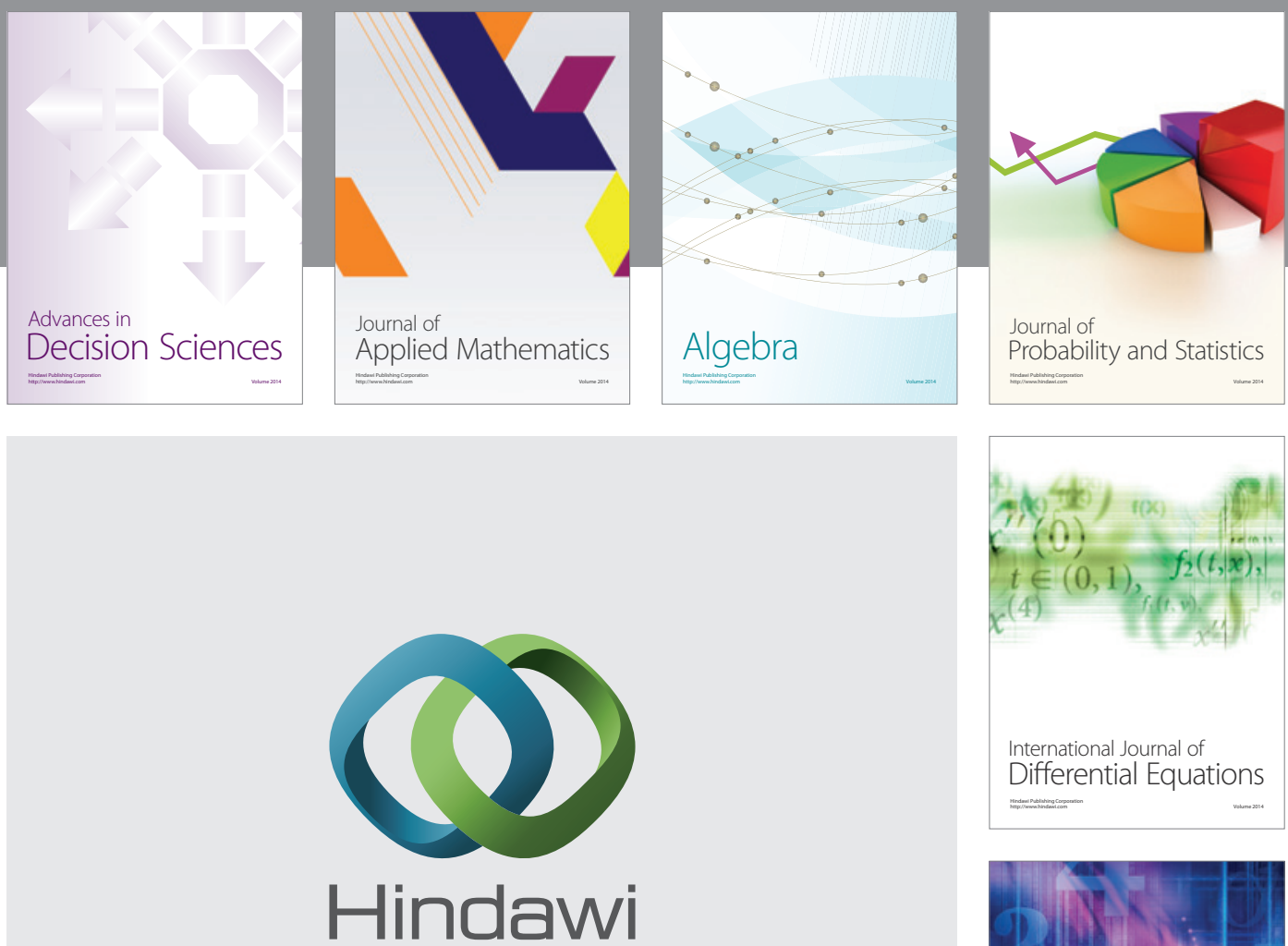

Submit your manuscripts at http://www.hindawi.com
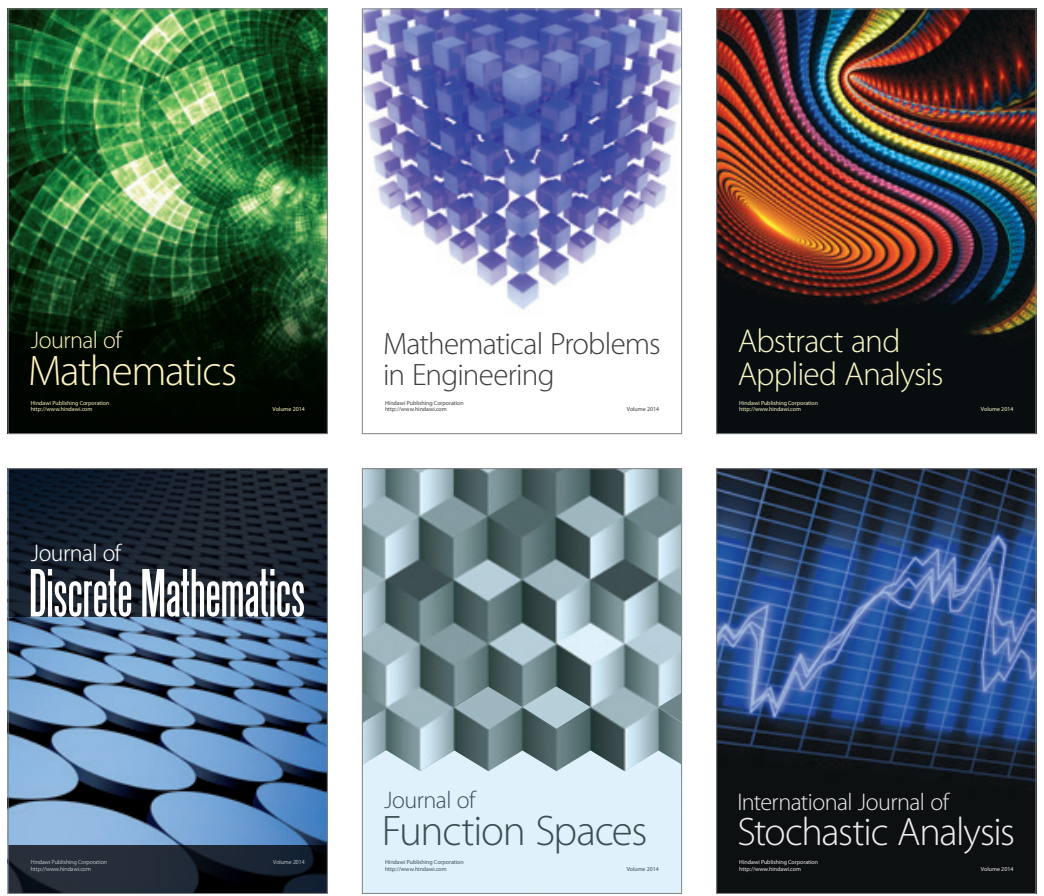

Journal of

Function Spaces

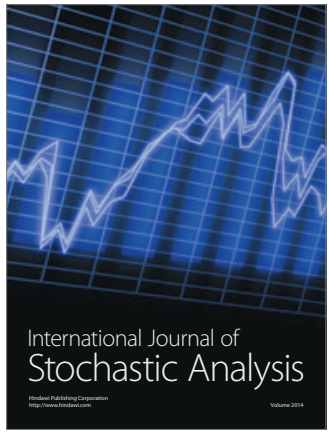

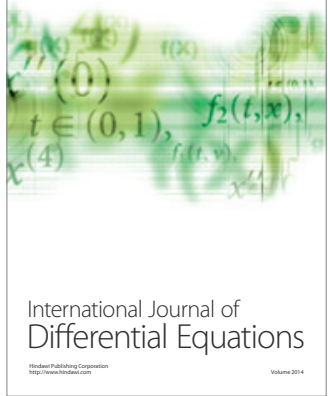
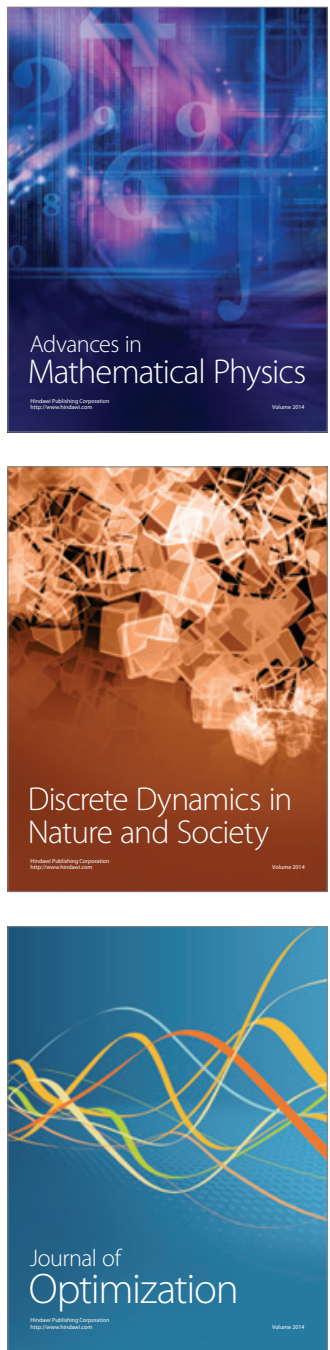